\title{
Self-assembled hierarchically structured organic-inorganic composite systems
}

\author{
Ulrich Tritschler ${ }^{1}$ and Helmut Cölfen \\ Physical Chemistry, University of Konstanz, Universitätsstraße 10, D 78457 Konstanz, Germany \\ 1 Present address: School of Chemistry, University of Bristol, Bristol BS8 1TS, UK. \\ E mail: helmut.coelfen@uni konstanz.de
}

Keywords: liquid crystal, anisotropic inorganic nanoparticles, liquid crystal polymer, bio inspired, organic inorganic composite materials/ hybrids, self organization, multifunctional

\begin{abstract}
Designing bio-inspired, multifunctional organic-inorganic composite materials is one of the most popular current research objectives. Due to the high complexity of biocomposite structures found in nacre and bone, for example, a one-pot scalable and versatile synthesis approach addressing structural key features of biominerals and affording bio-inspired, multifunctional organic-inorganic composites with advanced physical properties is highly challenging. This article reviews recent progress in synthesizing organic-inorganic composite materials via various self-assembly techniques and in this context highlights a recently developed bio-inspired synthesis concept for the fabrication of hierarchically structured, organic-inorganic composite materials. This one-step self-organization concept based on simultaneous liquid crystal formation of anisotropic inorganic nanoparticles and a functional liquid crystalline polymer turned out to be simple, fast, scalable and versatile, leading to various (multi-)functional composite materials, which exhibit hierarchical structuring over several length scales. Consequently, this synthesis approach is relevant for further progress and scientific breakthrough in the research field of bio-inspired and biomimetic materials.
\end{abstract}

Creating new, technologically relevant materials with a broad range of potential applications in materials science and the life sciences is a central goal for materials syntheses. Within this area, the fabrication of bio-inspired organic-inorganic composite materials has become increasingly popular in recent years [1-3]. Therefore, reproducing the complex structures of natural organic-inorganic composites by means of a scalable, versatile and simple synthesis concept is currently subject of intense research.

Biominerals consist of stiff and brittle mineral crystals that are joined by soft and ductile organic biomacromolecules. Prominent examples include nacre and bone [3, 4]. These biomaterials are well-known for their outstanding mechanical performance combining both high stiffness and toughness [5, 6]. Biominerals exhibit a fracture resistance which by far exceeds the one of the respective pure inorganic component. For instance, the fracture resistance of nacre, which consists of $95 \%$ of the $\mathrm{CaCO}_{3}$ polymorph aragonite, is 3000 times higher than the fracture resistance of pure aragonite [4]. The high fraction of inorganic crystals is the load-bearing element providing stiffness to the material while the organic phase surrounding the inorganic particles plays a decisive role for the toughness of the material $[7,8]$. Two structural characteristics contribute essentially to the exceptional mechanical performance of biominerals: a sophisticated hierarchical structure of organic and inorganic components and a well-controlled coupling at the interface of both constituents $[5,6]$.

Bone is a well-studied biomineral. On the lower hierarchical level, ranging from the nanometer length scale up to the scale of tens of micrometers, bone consists of highly ordered, staggered arrays of hydroxyapatite mineral platelets with a thickness of 2-4 nm embedded in collagen fibers (scheme 1) $[6,12]$. This oriented arrangement of aligned inorganic nanoplatelets within the organic matrix is called mesocrystal, which is a (3D) superstructure of nanocrystal building units with common crystallographic orientation [9-11]. The organic collagen matrix itself is hierarchically structured on several levels since the collagen fibrils are joined together to fiber bundles that 


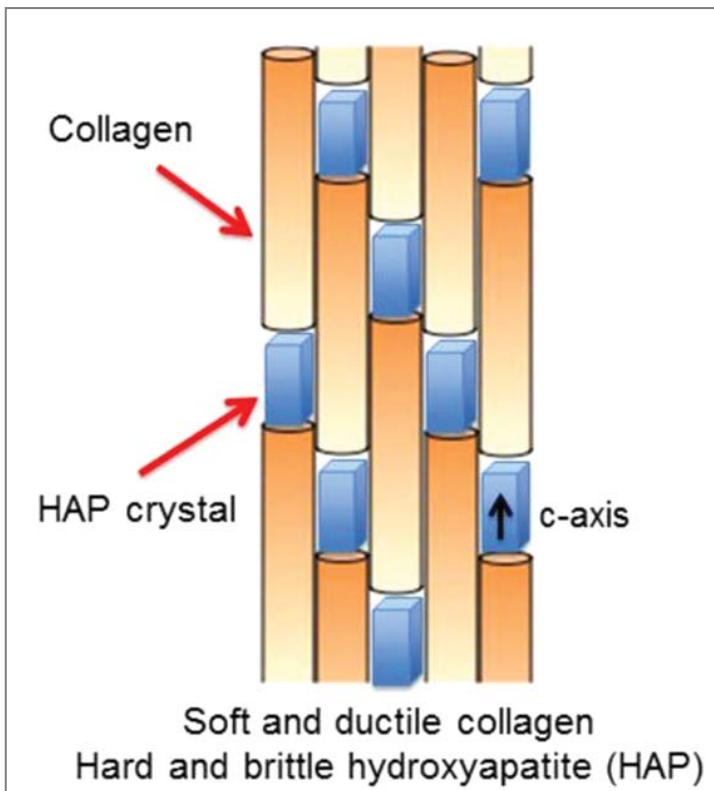

Scheme 1. Schematic representation of the structure of bone on the lowest hierarchical level: arrangement of hydroxyapa tite platelets in collagen triple helices.

finally form a lamellar structure [6, 12]. Proteoglycans may function as gluing components sticking the structural constituents together [8]. Both the staggered arrangement of apatite platelets within the collagen matrix and the tight binding between organic and inorganic phase are important features of this biomineral, allowing for an optimum combination of stiffness and toughness and different toughening mechanisms $[5,7,13,14]$. Moreover, the nanometersized particles provide an optimum strength and maximum tolerance of flaws (robustness) of this biomineral [15]. This biomineral archetype reveals that a successful approach for mimicking and reproducing biological structures needs to address (a) the hierarchical structuring of both organic and inorganic components, (b) a controlled coupling at the interface of both constituents, (c) a high loading of inorganic nanoparticles exhibiting a highly ordered arrangement, and (d) the construction of the ordered structures starting on the nanometer length scale.

Besides superior mechanical properties, the sophisticated hierarchical structure of biominerals can also lead to outstanding optical and magnetic properties. Amongst others, examples of these features include the optical iridescence of nacre and the (responsive) magnetic properties in magnetotactic bacteria [16-20].

Different concepts for the synthesis of bioinspired organic-inorganic lamellar composite materials via self-assembly techniques

In the past years, different bottom-up self-assembly concepts for mimicking the hierarchical structure and outstanding physical properties of biominerals have been developed [3]. Self-assembly processes may allow for precisely controlling structural dimensions on the nano- and/or micrometer length scale from the early synthesis stages and for introducing functionality into the material [21-24]. In particular, reproducing the highly ordered structure and remarkable mechanical and optical properties of nacre has been in the focus of many research groups, which led to a wide range of bio-inspired lamellar composite structures, mainly by using micro- and nanoplatelets, such as natural and synthetic clay minerals (montmorillonite and Laponite clay platelets) or alumina platelets. The fabrication of bio-inspired composite structures and the achieved mechanical properties of these composites have been very recently reviewed by Wegst and Ritchie et al [25], and therefore, only synthesis techniques relevant for the context of this paper will be summarized shortly.

An example for an early synthesis of montmorillonite clay/polymer hybrid materials is the work of Kamigaito et al [26]. They polymerized $\varepsilon$-Caprolactam in the interlayer of montmorillonite after cation exchange with 12-aminolauric acid which served as a polymerization initiator. This led to the formation of hybrid materials composed of nylon 6 and montmorillonite clay platelets, exhibiting uniformly distributed clay silicate layers within the nylon 6 phase. In addition, interactions between the nylon 6 matrix and the clay platelets via the ammonium cations of the polymer and the anions of montmorillonite were suggested.

Nacre-mimicking lamellar composite structures with remarkable physical properties were successfully fabricated via a layer-by-layer assembly technique [27-37]. Notable in this context, for example, is the work by Tang et al who used anionic montmorillonite clay platelets and the polycation poly(diallyldimethlyammonium) chloride to sequentially build up a clay/polyelectrolyte multilayered composite that resembles the structure of nacre. The composite material exhibits mechanical properties close to the mechanical performance of nacre, in terms of tensile strength, and bone, in terms of Young's modulus [31]. A high level of structural organization of sequentially fabricated poly(vinyl alcohol)/montmorillonite composites was found to lead to an efficient load transfer between the polymeric phase and the montmorillonite platelets [34]. Multilayered composite films with high tensile strength and ductile behavior were obtained by sequential deposition of colloidally assembled alumina platelets and chitosan. The alumina platelets were aligned at the air-water interface before transferring the 2D layered structure onto a substrate and subsequently, the chitosan layer was deposited by spincoating [32]. Finnemore et al successfully fabricated a multilayered material with a nacre-like structure consisting of $\mathrm{CaCO}_{3}$ platelets and porous organic layers via layer-by-layer deposition. This nacre imitation 


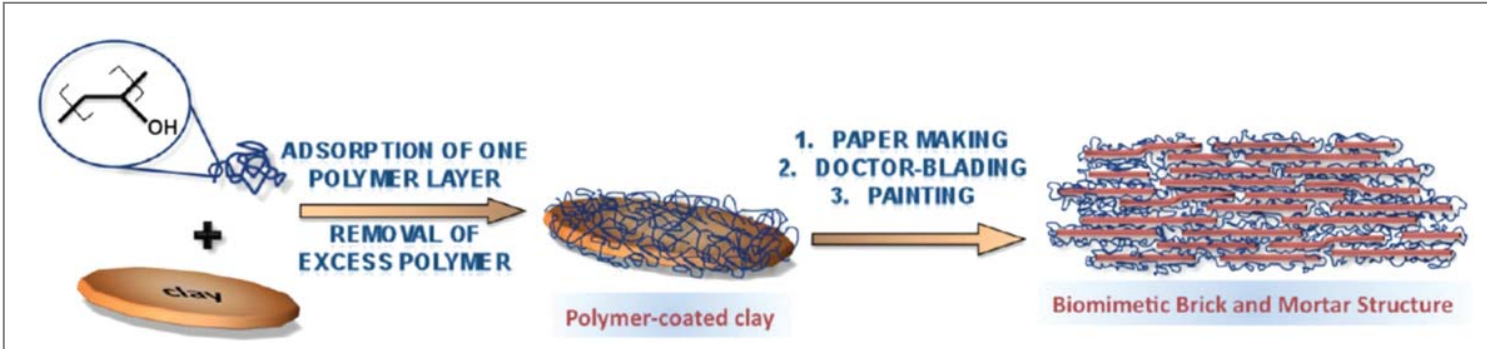

Scheme 2. Synthesis of biomimetic composite materials composed of montmorillonite clay platelets and polyvinylalcohol according to Ikkala et al [43]. Reproduced with permission from the American Chemical Society.

exhibited a nacre-like mechanical toughness and resembled nacre's optical iridescence [27].

Bio-inspired (multifunctional) composite films were obtained by applying solution-casting procedures. Transparent, flexible and solvent resistant composite films with a disco-nematic liquid crystalline structure of Laponite platelets and an amorphous polyethylene glycol matrix were obtained by Shikinaka et al [38]. For preparing these films, Laponite was modified with triethoxysilane modified polyethylene glycol via a sol-gel reaction and subsequently, an aqueous dispersion of the organically modified Laponite was dropped onto a suitable surface. Li et al [39] fabricated nacre-like layered composite films consisting of reduced poly(vinyl alcohol) and graphene oxide via a solution casting method. The reduction of the poly (vinyl alcohol)/graphene oxide composite films was performed by immersing the films into a hydroiodic acid solution at room temperature. The multifunctional, biocompatible films combine excellent mechanical and electrical properties.

Microcomposite materials which resemble the cross-lamellar microarchitecture of molluscan shells were produced by Ballarini et al [40] using microelectromechanical systems technology. This technique benefits from using photolithography to generate defined shapes. Bio-inspired composites were obtained by using silicon and photoresist. While the latter one was selected to mimic the tough organic matrix, silicon was used to introduce hardness into the material as known from aragonite in molluscan shells. These composites featured several energy-dissipating crack pattern characteristics and exhibited a remarkable strength and work of fracture [40].

Bio-inspired ceramic-based materials with high yield strength and fracture toughness were obtained by ice-templating and sintering [41, 42]. Layered ceramic porous scaffolds for the preparation of bio-inspired $\mathrm{Al}_{2} \mathrm{O}_{3} /$ poly(methyl methacrylate) materials were obtained from initial lamellar structures which were fabricated by freeze casting. To this end, the lamellar structures were infiltrated with a suitable polymer and subsequently uniaxially pressed and densified. After thermal treatment and sintering at $1500{ }^{\circ} \mathrm{C}$, the 'brick-and-mortar' scaffold was obtained. Finally, the porous substrate was infiltrated with poly(methyl methacrylate) via in situ free radical polymerization [41]. By applying this technique, Munch et al [41] successfully fabricated hybrid materials with the highest fracture toughness reported hitherto, even outperforming the fracture toughness of nacre.

Obviously, a challenging issue of current nacremimicking methods is their efficiency for up-scaling to yield bulk materials in a single synthesis step. Sequential deposition techniques are time-consuming as they require numerous synthesis steps to form composite materials with a thickness on the higher micrometer to millimeter scale, while ice-templating and sintering techniques demand considerable energy input to fabricate composites on a larger scale [41]. Recently, these hurdles have been managed to overcome and nacre-like composites have been synthesized via doctor-blading, paper-making or painting technique of a dispersion consisting of polyvinylalcohol adsorbed montmorillonite clay platelets (see scheme 2) [43]. These self-assembly methods are time-efficient, robust, and have the potential for upscaling. In addition, they allow for fabricating bioinspired clay/polymer composites with advanced mechanical properties (partially exceeding nacre) as well as excellent gas barrier and shape-persistent, fireretardant properties [43]. In a subsequent study, Ikkala et al [44] tailored the mechanical properties of the clay/polymer composites by changing the connectivity between the polymeric shells which surround the montmorillonite platelets, i.e. by replacing the covalent bonds between the montmorillonite/poly (vinyl alcohol) building blocks which originate from covalently cross-linking the poly(vinyl alcohol) chains [43] by ionic supramolecular bonds, using the polycation poly(diallyldimethyl-ammonium chloride for coating the clay platelets. The change of the binding motif introduces dynamic properties into the material and is an important characteristic in biological materials. By increasing the valency of the counterion, i.e. by exchanging the chloride ion for bi- and trivalent ions $\left(\mathrm{SO}_{4}^{2-}, \mathrm{PO}_{4}^{3-}\right)$, the physical cross-linking within the polymer layers can increase, leading to an increase of the stiffness and strength of the composite materials [44].

However, a one-pot scalable synthesis strategy to fabricate hierarchically organized composite materials 


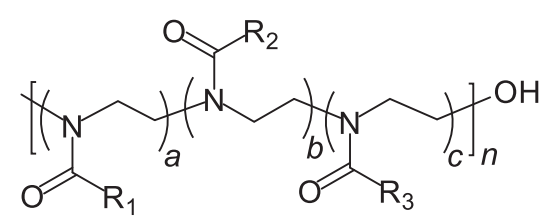

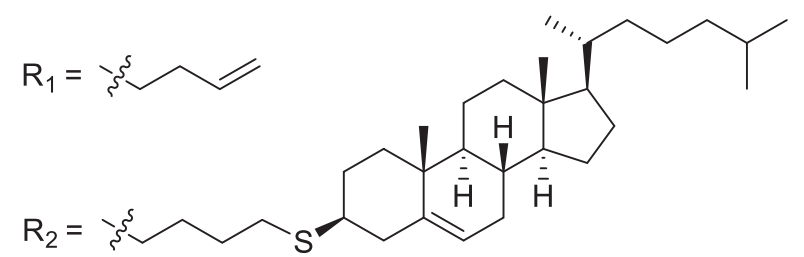<smiles>[R5][Y][14CH2]CCCSCCC(=O)O</smiles>

Scheme 3. Poly[2 (3 butenyl) 2 oxazoline] was synthesized via cationic ring opening polymerization of 2 (3 butenyl) 2 oxazoline and modified with thiocholesterol (Chol SH; liquid crystal unit) and 3 mercaptopropionic acid ( $3 \mathrm{MPA}$; binding/gluing unit) via thiol ene chemistry, leading to the statistical LC 'gluing' copolymer. The degree of polymerization was $n \sim 70 \quad 120$ and the degree of cholesterol and carboxyl functionalization ca. $20 \%$ and $30 \% 40 \%$, respectively [ [1 55$]$.

with a structured organic and inorganic phase as well as a glue between organic and inorganic components, all important features found in biocomposites, was still a major challenge in bio-inspired materials research. It is also of particular importance that the synthesis strategy is not only restricted to a single system example. The targeted strategy shall be rather versatile in order to apply it to other, technologically important inorganic particle systems with the aim of creating a wide range of multifunctional composites using the same underlying principle. For designing technologically relevant organic-inorganic composites, functional nanoparticles, such as vanadium pentoxide $\left(\mathrm{V}_{2} \mathrm{O}_{5}\right)$, can be used. For example, $\mathrm{V}_{2} \mathrm{O}_{5}$ mesocrystals formed by adding a surface-active polymer to a $\mathrm{V}_{2} \mathrm{O}_{5}$ dispersion consisting of pre-oriented nanoparticle building units $\left(\mathrm{V}_{2} \mathrm{O}_{5}\right.$ tactoid $)$ [45].

\section{Bio-inspired liquid crystal based fabrication concept for organic-inorganic composites}

The synthesis of bio-inspired organic-inorganic composites involving the use of liquid crystals as templates was reported. For example, Kato et al [46] used chitin as a template for the crystallization of calcium carbonate. This template crystallization method within an ordered, nematic LC chitin matrix led to the formation of unidirectionally aligned hybrids and resembled the structure formation of the exoskeleton of the jewel beetle. Very recently, they succeeded in synthesizing a homogeneous hybrid structure composed of chitin and calcium carbonate that exhibits a helical ordering and resembles the composition as well as the structure of the exosceleton of crustaceans [47]. In this study, they used LC chitin whiskers that act as helically ordered templates stabilized by acidic polymer networks and initiated the calcium carbonate crystallization with amorphous calcium carbonate precursors. An approach for the formation of chiral organic-inorganic superstructures via a self-assembly pathway without using a precursor template was reported by Yu et al [48]. For this system a racemic double hydrophilic block copolymer containing a block that interacts with the inorganic compound and a second non-interacting block was used. Achiral barium carbonate nanocrystals were found to align helically in presence of this block copolymer, induced by phase-selective polymer adsorption to the nanocrystals.

Self-organization of polymers via LC formation was shown to be able to mimic the structural and optical properties of the golden beetle Chrysina Resplendens [49]. Reactive mesogens used in this study keep their conformation after photopolymerization. Selfassembly via liquid crystal formation is a crucial concept applied by nature for many biological systems [50]. And recent studies [46, 47] revealed that LC formation is a highly promising technique for the synthetic re-production of bio-inspired organic-inorganic hybrid structures exhibiting highly defined and ordered structures.

Very recently, a bio-inspired scalable synthesis concept to fabricate organic-inorganic composite materials via a scalable one-step self-organization process was developed. This concept is based on simultaneous LC formation of organic and inorganic components, i.e. structuring of both organic and inorganic phases, induced by shearing the initially prepared organic-inorganic hybrid particle dispersion. The organic matrix is a poly(2-oxazoline), more exactly poly[2-(3-butenyl)-2-oxazoline], functionalized with pendant cholesteryl and carboxyl groups (statistical LC 'gluing' copolymer; see scheme 3) [51].

The cholesteryl side groups allow the statistical LC 'gluing' copolymers to form lyotropic chiral nematic phases, and upon shearing, these side groups align to form lyotropic phases on the length scale of up to several hundreds of micrometers [51]. The carboxyl functionality enables the polymer to selectively bind ('glue') to anisotropic nanoparticles via electrostatic 


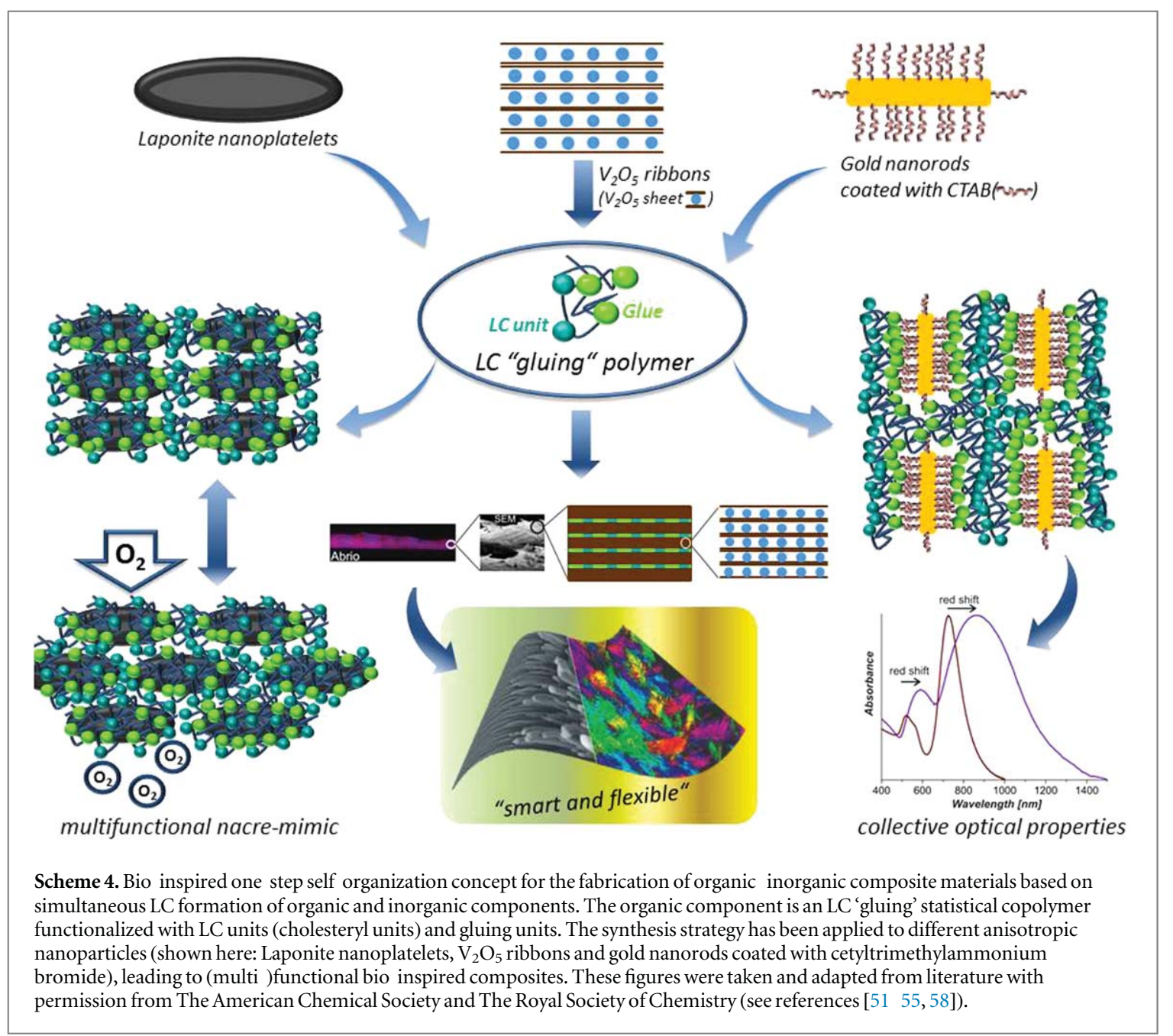

interaction [51, 55] or hydrogen bonding [54]. In a typical experiment, a dispersion of suitable nanoparticles was combined with a dispersion or solution of the LC 'gluing' copolymer under defined conditions to allow for binding between the nanoparticles and the polymer chains and thus, for forming hybrid particles. If required, phase-transfer of the hybrid particles into a suitable organic solvent was performed, enabling the cholesteryl side chains to form lyotropic phases. Selfassembly of the polymer may guide the nanoparticle positioning and alignment. This is additionally supported by the anisotropic shape of the nanoparticles, which enables the particles to form an LC phase. Shearing was applied to induce long-range ordering of both the organic phase and inorganic phase simultaneously [51-55].

By using Laponite [56] nanoplatelets, nacremimicking materials structured on several levels of hierarchy were developed (see scheme 4 , left panel) $[51,52]$. The LC 'gluing' copolymers are able to selectively bind to the positively charged rims of the Laponite platelets due to their negatively charged carboxyl functionalities under basic conditions, leading to Laponite/LC polymer hybrid particles. After exfoliating the hybrid material in a suitable organic medium, in which the cholesteryl side groups are able to form lyotropic phases, the dispersion of hybrid particles was sheared to induce simultaneous self-organization of organic and inorganic components (figure 1) [51, 52].

In dry state, the composites exhibit a hierarchical structuring on the scale of several hundreds of micrometers with a pronounced layered structure in horizontal dimension [51, 52]. The nanostructure was found to be highly dependent on the polymer fraction. Laponite/LC polymer composites consisting of a ratio of Laponite/LC polymer $=1: 1$ and 2:1 w/w exhibit a columnar arrangement of Laponite platelets within the structured organic matrix forming mesocrystalline arrangements [51], and resembling the structure of columnar nacre. By lowering the polymer fraction (e.g. Laponite/LC polymer $=5: 1 \mathrm{w} / \mathrm{w}$ ), Laponite/LC polymer composites with a brick-and-mortar structure were formed, exhibiting a platelet nanostructure that does not possess cores and overlap regions, similar to the one found for sheet nacre [52]. In addition to optical anisotropy, the Laponite/LC polymer composites exhibit anisotropic mechanical properties with values for reduced modulus and hardness comparable to those found in human femur $[51,52,57]^{2}$.

${ }^{2}$ Nanoindentation measurements of reference [57] were performed in the wet state. 


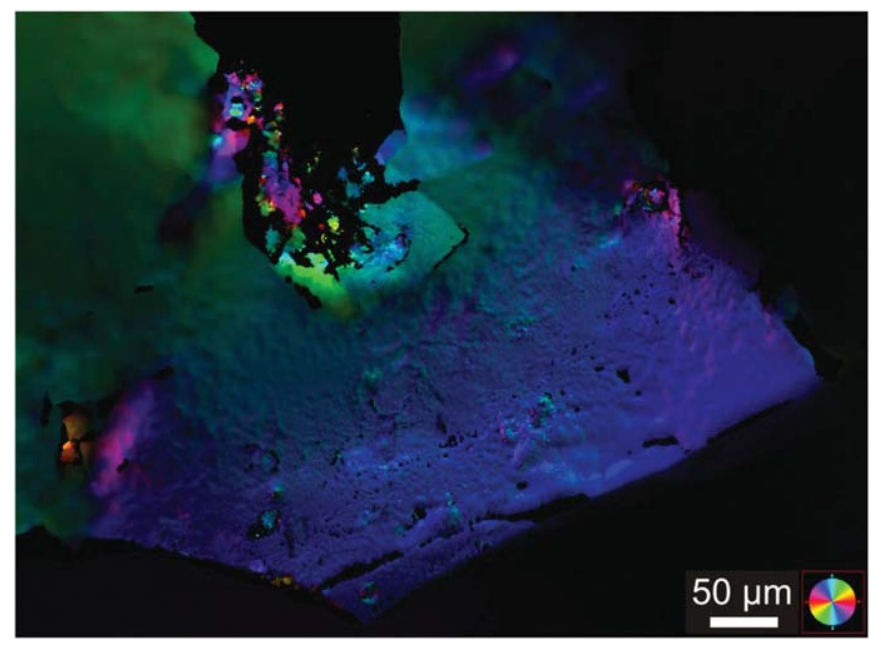

Figure 1. Top view of a representative quantitative birefringence microscopy (Abrio) image of a shear induced Laponite/LC polymer composite in dry state (here shown a dried Laponite/LC polymer 5:1 w/w composite obtained after rotational shearing by means of a shear cell). The color saturation indicates the degree of structural orientation, which is illustrated by the color wheel (bottom right).

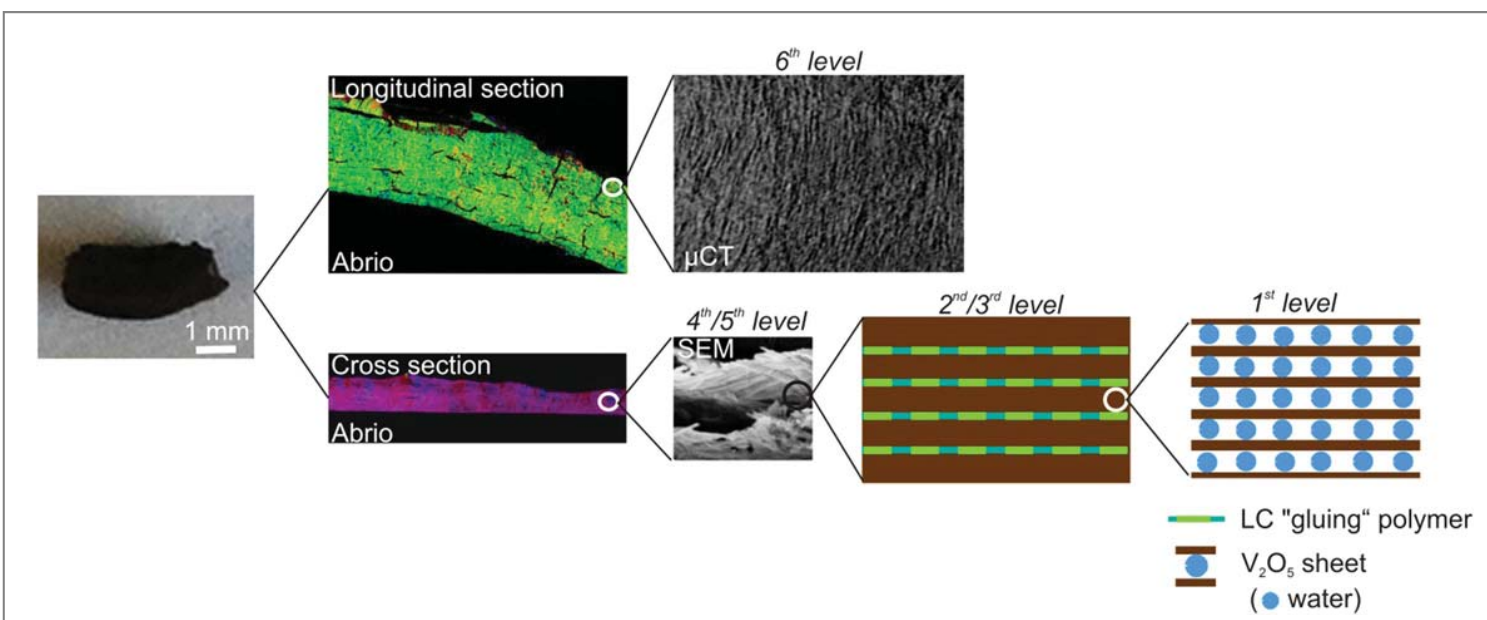

Scheme 5. Hierarchical composite structure composed of vanadium pentoxide and LC polymer (adapted from reference [54] and reproduced with the permission of the American Chemical Society). The six hierarchical levels are indicated as headings of the respective images.

Furthermore, Laponite/LC polymer composite coatings of different ratios were very recently shown to possess advantageous gas barrier properties, rendering this composite system multifunctional (see scheme 4, left panel) [52].

In addition, this simple, fast, modular and scalable concept for the fabrication of bio-inspired hierarchically structured composites has been proven to be a fairly universal tool. Anisotropic nanoparticles of different shape and surface functionalization/modification were self-assembled using the statistical LC 'gluing' copolymer (see scheme 4).

By applying the fabrication concept to $\mathrm{V}_{2} \mathrm{O}_{5}$ [59-62] ribbons, hierarchically structured multifunctional $\mathrm{V}_{2} \mathrm{O}_{5} / \mathrm{LC}$ polymer composites organized on six levels of hierarchy from the higher micrometer down to the nanometer scale were synthesized (see scheme 4, middle panel and scheme 5) [54]. Binding between $\mathrm{V}_{2} \mathrm{O}_{5}$ ribbons and the LC polymer was suggested to occur through hydrogen bridges via the oxygen-terminated $\mathrm{V}_{2} \mathrm{O}_{5}$ particle faces and the protonated carboxyl groups of the polymer. The aligned $\mathrm{V}_{2} \mathrm{O}_{5}$ ribbons, which are composed of selfassembled $\mathrm{V}_{2} \mathrm{O}_{5}$ sheets, are surrounded by lyotropic polymeric phases. Further structural features on the nanometer to higher micrometer length scale of these hierarchically organized composites include a pronounced layered structure in horizontal dimension and a textured substructure in vertical dimension (scheme 5). These highly defined structures (mesocrystalline arrangements) were obtained when preparing the composites with both an initial pre-oriented $\mathrm{V}_{2} \mathrm{O}_{5}$ dispersion $\left(\mathrm{V}_{2} \mathrm{O}_{5}\right.$ tactoid $)$ and an initial isotropic $\mathrm{V}_{2} \mathrm{O}_{5}$ dispersion [54]. These composite materials combine enhanced mechanical and advantageous electrochromic properties, making it an interesting 
candidate for novel technological applications such as 'smart and flexible windows' [53]. The anisotropy in mechanical properties of the $\mathrm{V}_{2} \mathrm{O}_{5} / \mathrm{LC}$ polymer composite materials determined by nanoindentation confirmed the structural anisotropy. The composite structure was found to influence the mechanical performance of the composites significantly. $\mathrm{V}_{2} \mathrm{O}_{5} / \mathrm{LC}$ polymer composites with a well-defined hierarchical structure exhibited mechanical properties comparable to biocomposites like human bone and dentin [54]. Furthermore, the $\mathrm{V}_{2} \mathrm{O}_{5} / \mathrm{LC}$ polymer composite films prepared via this one-step self-organization and costefficient process featured advantageous electrochromic properties, changing their color under alternating potentials. Lithium intercalation into $\mathrm{V}_{2} \mathrm{O}_{5}$ was induced by applying a cathodic potential, leading to a bleaching of the initial yellow, thin composite film. Upon subsequent lithium extraction by applying an anodic potential, the yellow color of the composite film was retrieved. These composite films benefit from a long-term cycle stability under alternating potentials and operate under normal conditions due to their resistance towards oxygen and moisture. Moreover, the composite structures on the nanometer to micrometer length scale remained visually intact after extended voltammetric cycling and storing the electrochromic devices for several weeks (see scheme 4 , middle panel) [53]. Since vanadium oxides are functional materials with a fairly broad range of applications including catalytic applications [63-68] and the use as gas sensors [69-72], hierarchically organized $\mathrm{V}_{2} \mathrm{O}_{5}$ /LC polymer composites (or their micro-) nanoporous counterparts obtained after removal of the polymeric matrix) might potentially even feature further promising functions.

Collective optical properties were addressed by applying the bio-inspired fabrication concept to gold nanorods coated with cetyltrimethylammonium bromide (CTAB) [55]. The carboxy side chains of the LC 'gluing' polymer are able to bind to the positively charged CTAB layer of the gold nanorods via electrostatic interaction. Upon shearing the gold nanorod/ LC polymer hybrid dispersion in DMF via spin-coating, composite films with high inorganic fractions $>80 \%$ were obtained, which exhibit a homogenous distribution of gold particles within the polymeric lyotropic matrix on the length scale of several tens of micrometers. The gold nanorods were locally aligned forming gold nanorod network assemblies with both end-to-end and side-by-side combinations. These network structures led to significant red-shifts of the longitudinal and transversal surface plasmon resonances compared to the respective resonances of the hybrid particles dispersed in solution (see scheme 4, right panel). Thus, control over collective optical properties was achieved by preparing bio-inspired, structured gold nanorod/LC polymer composite films with high inorganic fractions via the polymer-directed self-organization concept. This merged two disparate research fields and gave rise to a large number of potential technical applications including bio-inspired optical filter systems. Also, this concept may potentially be applied to different other optically active, anisotropic nanoparticle systems to tune their collective optical properties [55].

In summary, a one-step self-organization concept for the fabrication of bio-inspired organic-inorganic composites based on LC formation of organic and inorganic components was developed, using an LC 'gluing' copolymer and anisotropic inorganic nanoparticles. This concept addresses key features of biological composite systems, in particular the binding between organic and inorganic constituents, the hierarchical organization of organic and inorganic components over several length scales starting on the nanometer scale, and the high loading of inorganic particles. In addition, this self-organization concept is simple, fast, scalable, and rather versatile regarding nature and morphology as well as surface charge and surface modification/functionalization of the anisotropic inorganic nanoparticles. By applying this synthesis strategy to different nanoparticle systems, a variety of functional and multifunctional hierarchically structured composite systems were fabricated, which cover well-tuned mechanical, optical and electrochromic properties (see scheme 4).

This strategy has the potential of designing binding motifs between the LC 'gluing' polymer and various inorganic nanoparticles. Suitable binding units for a specific nanoparticle system can be found by using the phage display technique. This technique has recently been shown to function as a powerful tool in the field of material sciences for screening binding motifs and determining effective organic adsorbing additives on inorganic nanoparticles (calcium silicate hydrate platelets) [73]. Adequate binding units can be easily introduced into the side chains of the LC 'gluing' polymer by applying thiol-ene photochemistry, revealing once more the versatile character of the bio-inspired onestep self-organization strategy for the synthesis of organic-inorganic composites.

The non-covalent glue between organic and inorganic components as well as the liquid crystal structure of the polymeric matrix might potentially give rise to self-healing properties. Furthermore, the non-covalent binding mode may possess the potential to be opened, stepwise and reversibly, upon applying mechanical stress on the composite material, thus functioning as 'sacrificial bonds'. Consequently, these 'hidden lengths' might prevent crack propagation within the composite material on the nano- to micrometer scale to a certain extent. These considerations give rise to advanced materials properties and are currently part of future studies.

The self-assembly of anisotropic particles coated with magnetic nanoparticles within a structured lyotropic polymeric matrix may be of potential interest in the context of orienting nanoparticles up to the 
macroscopic scale by means of an external magnetic field as well as re-orienting them to repair defects formed upon applying mechanical stress. The concept of controlling the orientation and distribution of magnetite coated, anisotropic microparticles within a polymeric matrix by means of an external magnetic field, leading to locally reinforced, wear-resistant composites, was reported by Studart et al [74]. Magnetically responsive, anisotropic particles were, for example, shown to act as a template to create materials with controlled anisotropic porosity, potentially of interest in tissue regeneration [75]. Most recently, the concept of using magnetically responsive particles to control their orientation and distribution by means of external magnetic fields was combined with a slipcasting process, leading to the so-called magnetically assisted slip casting process. This strategy allowed the fabrication of multilayered, bio-inspired heterogeneous composites with complex-shaped microstructures and high inorganic fractions [76]. The concept of using particles coated with magnetic nanoparticles for controlling the orientation and distribution up to the micro- to millimeter scale might also be promising for the bio-inspired, one-step self-organization concept based on LC formation, in particular in the context of creating self-healing composites or stimuli-responsive composites featuring defined microand nanostructures that can be switched between an ordered state and a disordered state upon applying appropriate external magnetic fields. In addition, applying the highlighted bio-inspired fabrication concept to anisotropic paramagnetic particles and inducing long-range orientation of these particles within the structured lyotropic polymeric matrix by means of an external magnetic field might lead to mechanically reinforced composites with new collective magnetic properties due to the mutual alignment of the magnetic nanoparticles.

The bio-inspired, LC driven concept might not only be limited to polyoxazoline-based polymers, but could be rather universal. Consequently, stimuli-responsive composites might be fabricated by using various other polymeric matrices, which potentially allow for controlling and reversibly changing the orientation of the compliant inorganic nanoparticles and thus, the physical properties of the composite material.

These are only some aspects for perspective research to expand the scope of this new, simple, timeefficient, scalable, and versatile bio-inspired structuring concept for fabricating organic-inorganic composites based on LC formation. Designing bio-inspired hierarchically structured materials which combine multiple functions, excellent physical properties and a stimuli-responsive behavior by using inexpensive, functional polymers and suitable inorganic nanoparticles is a highly competitive and challenging current and future research objective for both academia and industry.

\section{Acknowledgments}

UT and HC thank the DFG priority program SPP1420 as well as BASF SE for their financial support. The highlighted work has been published in different journal articles (see reference section) and in the $\mathrm{PhD}$ thesis of UT.

\section{References}

[1] Meyers M A, McKittrick J and Chen P Y 2013 Science 339 7739

[2] Rieger J, Kellermeier M and Nicoleau L 2014 Angew. Chem., Int. Ed. 531238096

[3] Meldrum F C and Cölfen H 2008 Chem. Rev. 1084332432

[4] Lowenstam H A and Weiner S 1989 On Biomineralization (New York: Oxford University Press)

[5] Fratzl P and Weinkamer R 2007 Prog. Mater. Sci. 521263334

[6] Aichmayer B and Fratzl P 2010 Phys. J. 9338

[7] Jäger I and Fratzl P 2000 Biophys. J. 79173746

[8] Fratzl P, Burgert I and Gupta H S 2004 Phys. Chem. Chem. Phys. 655759

[9] Bergström L, Sturm E V, Salazar Alvarez G and Cölfen H 2015 Acc. Chem. Res. 481391402

[10] Cölfen H and Antonietti M 2008 Mesocrystals and Nonclassical Crystallization (New York: Wiley)

[11] Cölfen H and Antonietti M 2005 Angew. Chem., Int. Ed. 44 557691

[12] Fratzl P, Gupta H S, Paschalis E P and Roschger P 2004 J. Mater. Chem. 14211523

[13] Ritchie R O 2011 Nat. Mater. 1081722

[14] Fantner G E et al 2005 Nat. Mater. 46126

[15] Gao H, Ji B, Jäger I L, Arzt E and Fratzl P 2003 Proc. Natl Acad. Sci. USA 1005597600

[16] Gur D, Leshem B, Pierantoni M, Farstey V, Oron D, Weiner S and Addadi L 2015 J. Am. Chem. Soc. 137840811

[17] Aizenberg J, Tkachenko A, Weiner S, Addadi L and Hendler G 2001 Nature 41281922

[18] Sundar V C, Yablont A D, Grazul J L, Ilant M and Aizenberg J 2003 Nature 424899

[19] Weiner S 2008 J. Struct. Biol. 16322934

[20] Faivre D and Schüler D 2008 Chem. Rev. 108487598

[21] Shimomura M and Sawadaishi T 2001 Curr. Opin. Colloid Interface Sci. 6116

[22] Nichol J W and Khademhosseini A 2009 Soft Matter 513129

[23] Seeman N C and Belcher A M 2002 Proc. Natl Acad. Sci. USA 99 64515

[24] Ozin G A 1992 Adv. Mater. 461249

[25] Wegst U G K, Bai H, Saiz E, Tomsia A P and Ritchie R O 2015 Nat. Mater. 142336

[26] Usuki A, Kojima Y, Kawasumi M, Okada A, Fukushima Y, Kurauchi T and Kamigaito O 1993 J. Mater. Res. 8117984

[27] Finnemore A, Cunha P, Shean T, Vignolini S, Guldin S, Oyen M and Steiner U 2012 Nat. Commun. 3966

[28] Podsiadlo P, Liu Z, Paterson D, Messersmith P B and Kotov N A 2007 Adv. Mater. 1994955

[29] Wei H, Ma N, Shi F, Wang Z and Zhang X 2007 Chem. Mater. 1919748

[30] Yeom B, Suhan K, Jinhan C, Junhee H and Kookheon C 2006 J. Adhes. 8244768

[31] Tang Z, Kotov N A, Magonov S and Ozturk B 2003 Nat. Mater. 24138

[32] Bonderer L J, Studart A R and Gauckler L J 2008 Science 319 106973

[33] Kato T 2000 Adv. Mater. 1215436

[34] Podsiadlo P et al 2007 Science 318803

[35] Podsiadlo P etal 2008 Nano Lett. 8176270

[36] Podsiadlo P, Michel M, Critchley K, Srivastava S, Qin M, Lee J W, Verploegen E, Hart A J, Qi Y and Kotov N A 2009 Angew. Chem., Int. Ed. 4870737 
[37] Podsiadlo P, Shim B S and Kotov N A Coord. Chem. Rev. 2009 253283551

[38] Shikinaka K, Aizawa K, Fujii N, Osada Y, Tokita M, Watanabe J and Shigehara K 2010 Langmuir 26124935

[39] Li Y Q, Yu T, Yang T Y, Zheng L X and Liao K 2012 Adv. Mater. 24342631

[40] Chen L, Ballarini R, Kahn H and Heuer A H 2007 J. Mater. Res. 2212431

[41] Munch E, Launey M E, Alsem D H, Saiz E, Tomsia A P and Ritchie R O 2008 Science 322151620

[42] Deville S, Saiz E, Nalla R K and Tomsia A P 2006 Science 311 5158

[43] Walther A, Bjurhager I, Malho J M, Pere J, Ruokolainen J, Berglund L A and Ikkala O 2010 Nano Lett. 1027428

[44] Walther A, Bjurhager I, Malho J M, Ruokolainen J, Berglund L and Ikkala O 2010 Angew. Chem., Int. Ed. 49 644853

[45] Lausser C, Cölfen H and Antonietti M 2011 ACS Nano 5 10714

[46] Nishimura T, Ito T, Yamamoto Y, Yoshio M and Kato T 2008 Angew. Chem., Int. Ed. 4728003

[47] Matsumura S, Kajiyama S, Nishimura T and Kato T 2015 Small 11512733

[48] Yu S H, Cölfen H, Tauer K and Antonietti M 2005 Nat. Mater. 4515

[49] Matranga A, Baig S, Boland J, Newton C, Taphouse T, Wells G and Kitson S 2013 Adv. Mater. 255203

[50] Rey A D and Herrera Valencia E E 2012 Biopolymers 97 37496

[51] Tritschler U, Zlotnikov I, Zaslansky P, Aichmayer B, Fratzl P, Schlaad H and Cölfen H 2013 Langmuir 2911093101

[52] Tritschler U, Zlotnikov I, Fratzl P, Schlaad H, Grüner S and Cölfen H 2016 Bioinspir. Biomim. (accepted for publication)

[53] Tritschler U, Beck F, Schlaad H and Colfen H 2015 J. Mater. Chem. C 39504

[54] Tritschler U, Zlotnikov I, Zaslansky P, Fratzl P, Schlaad H and Cölfen H 2014 ACS Nano 85089104

[55] Tritschler U, Zlotnikov I, Keckeis P, Schlaad H and Cölfen H 2014 Langmuir 301378190
[56] BYK Additives \& Instruments, Laponite Performance Additives, Technical Brochure available online at http://byk. com/en

[57] Zysset P K, Edward Guo X, Edward Hoffler C, Moore K E and Goldstein S A 1999 J. Biomech. 32100512

[58] Tritschler U, Beck F, Schlaad H and Cölfen H J. Mater. Chem. C 20153902902

[59] Livage J 1998 Coord. Chem. Rev. 178809991018

[60] Davidson P, Batail P, Gabriel J C P, Livage J, Sanchez C and Bourgaux C 1997 Prog. Polym. Sci. 2291336

[61] Livage J 1991 Chem. Mater. 357893

[62] Petkov V, Trikalitis P N, Bozin E S, Billinge S J L, Vogt T and Kanatzidis M G 2002 J. Am. Chem. Soc. 1241015762

[63] Schimmoeller B, Schulz H, Pratsinis S E, Bareiss A, Reitzmann A and Kraushaar Czarnetzki B 2006 J. Catal. 243 8292

[64] Legrouri A, Baird T and Fryer J R 1993 J. Catal. 14017383

[65] Nikolov V, Klissurski D and Anastasov A 1991 Catal. Rev. Sci. Eng. 3331974

[66] Ramirez R, Casal B, Utrera L and Ruizhitzky E 1990 J. Phys. Chem. 9489605

[67] Wainwright M S and Foster N R 1979 Catal. Rev. Sci. Eng. 19 21192

[68] Colpaert MN 1973 Z. Phys. Chem. 841506

[69] Mao C J, Pan H C, Wu X C, Zhu J J and Chen H Y 2006 J. Phys. Chem. B 1101470913

[70] Biette L, Carn F, Maugey M, Achard M F, Maquet J, Steunou N, Livage J, Serier H and Backov R 2005 Adv. Mater. 1729704

[71] Liu J, Wang X, Peng Q and Li Y 2005 Adv. Mater. 177647

[72] Raible I, Burghard M, Schlecht U, Yasuda A and Vossmeyer T 2005 Sensor. Actuat. B 1067305

[73] Picker A, Nicoleau L, Nonat A, Labbez C and Cölfen H 2014 Adv. Mater. 26113540

[74] Erb R M, Libanori R, Rothfuchs N and Studart A R 2012 Science 335199204

[75] Sommer M R, Erb R M and Studart A R 2012 ACS Appl. Mater. Interfaces 4508691

[76] Le Ferrand H, Bouville F, Niebel T P and Studart A R 2015 Nat. Mater. 1411729 